\title{
Lesionia: a digital data management system for epidemiological and clinical data collected from patients suspected for cutaneous leishmaniasis
}

Emna Harigua ( $\nabla$ harigua.emna@gmail.com )

Institut Pasteur de Tunis https://orcid.org/0000-0003-2974-9157

Youssef Ben Salem

Universite de Carthage Ecole Superieure de Statistique et de l'Analyse de l'Information

Maaoui Hariga

Universite de Tunis El Manar Institut Superieur d'Informatique

Yusr Saadi Ben Aoun

Institut Pasteur de Tunis

Hajer Souguir

Institut Pasteur de Tunis

Hamed Chouaib

Institut Pasteur de Tunis

Imen Mkada

Institut Pasteur de Tunis

Zeineb Moussa

Institut Pasteur de Tunis

Akila Fathallah-Mili

Institut Pasteur de Tunis

Meryem Lemrani

Institut Pasteur du Maroc

Nabil Haddad

University of Public Health, Beirut

Oussama Souiai

Institut Pasteur de Tunis

Insaf Bel Hadj Ali

Institut Pasteur de Tunis

Ikram Guizani

Institut Pasteur de Tunis 
Keywords: Digital data management, database, cutaneous leishmaniasis, clinical data

Posted Date: February 3rd, 2020

DOI: https://doi.org/10.21203/rs.2.22461/v1

License: (c) (1) This work is licensed under a Creative Commons Attribution 4.0 International License. Read Full License 
Title: Lesionia: a digital data management system for epidemiological and clinical data collected from patients suspected for cutaneous leishmaniasis

Authors: Emna Harigua-Souiai*(1), Youssef Ben Salem†(1,2), Maaoui Hariga $†(1)$, Yusr Saadi(1), Hejer Souguir $\ddagger(1)$, Hamed Chouaieb $\ddagger(1,3)$, Imen Mkada(1), Zeineb Moussa(1), Akila Fathallah-Mili(1,3), Meryem Lemrani(4), Nabil Haddad (5), Oussama Souiai(6), Insaf Bel Hadj Ali(1), Ikram Guizani(1).

$\dagger$ Equal contributors: YBS \& MH

$\ddagger$ Equal contributors: HS \& HC

* Corresponding author: harigua.emna@gmail.com ; emna.harigua@pasteur.utm.tn

\section{Affiliations:}

(1) Laboratory of Molecular Epidemiology and Experimental Pathology - LR16IPT04, Institut Pasteur de Tunis, Université de Tunis El Manar, Tunis, Tunisia.

(2) École Supérieure de la Statistique et de l'Analyse de l'Information de Tunis, Université de Carthage, Tunisia.

(3) Parasitology Department, Hospital Farhat Hachad, Faculté de Médecine de Sousse, Tunisia.

(4) Laboratory of Parasitology and Vector-Borne-Diseases, Institut Pasteur du Maroc, Casablanca, Morocco.

(5) University of Public Health, Beirut, Lebanon.

(6) Laboratory of Bioinformatics, Mathematics and Statistics LR16IPT09, Institut Pasteur de Tunis, Université de Tunis El Manar, Tunis, Tunisia. 


\begin{abstract}
Background. Digital systems for data management (DSDM) are considered nowadays of high importance in the field of biomedical sciences. Such systems ensure that data meet the standards of FAIR (Findability, Accessibility, Interoperability and Reusability). Our group is interested in implementing a DSDM for data collected from patients suspected of having cutaneous leishmaniasis (CL) in the frame of diagnostics evaluation. The data is collected in multiple sites and countries by different partners in the frame of a project supported by the USAID-NAS PEER program. We capitalized on the thorough clinical and field expertise of some partners to assess needs. Then, we further refined these needs consortium-wide to define the data to be collected by the clinicians and biologists during the data life cycle. This led to the development of a questionnaire form for data collection and the implementation of a web-based application, called Lesionia.
\end{abstract}

Results. Based on the questionnaire, we developed Lesionia, a digital system for the management and the analysis of clinical and epidemiological data. It consists of a relational database and a web-based user interface (WUI). The database was conceived to be expandable to new collaborators and projects. It allows for data handling from the consented patient interview and sample collection to the samples storage and investigation. The WUI permits data entry, fetching, visualization and analysis. Rigorous controls on data entry were implemented to reduce discrepancies. It also offers a set of analysis tools that range from descriptive statistics to variable correlation analysis. Lesionia is accessible in a secure manner to all users of the consortium through a web browser connected to the Internet.

Conclusion. Lesionia is a valuable tool for clinical and epidemiological data management. It is an open source software that can broadly serve the scientific community interested in studying, controlling, reporting and diagnosing CL and similar cutaneous diseases.

Key words: Digital data management, database, cutaneous leishmaniasis, clinical data 


\section{Background}

Leishmaniasis is a group of largely distributed vector-borne parasitic diseases, endemic in more than 98 countries worldwide. Cutaneous leishmaniasis (CL) is considered by the World Health Organization (WHO) as one of the most neglected tropical diseases (NTD). It is a group of cutaneous diseases with high morbidity rates (https://www.who.int/leishmaniasis/en/ ). CL are associated with illiteracy, gender discrimination, weakness of the immune system and lack of resources amongst other factors and they inflict a major social burden (Bennis et al., 2018). Nonetheless, there is an underestimation of CL cases in endemic regions, due to diagnosis and reporting issues (Bailey et al., 2017), including countries of the MENA region and sub-saharan Africa (Alam et al., 2016; Tabbabi, 2019). Available data on CL global occurrence are sparse (Pigott et al., 2014), which presents an impediment to evaluating the disease burden and to the implementation of control strategies (Bailey et al., 2017).

More broadly, epidemiological studies of CL often lack robust and high quality data, as for most of NTDs (Ali et al., 2006, Jajosky et al., 2004). Implementing digital systems for data management (DSDM) appeared as part of the solution for a better quality of data in the case of Malaria and Dengue fever (Einsen et al., 2011; Thomsen et al., 2016), as well as studying the spread of Schistosoma japonicum in China (Gray et al., 2009). Introducing DSDM was assessed on different types of studies ranging from geo-spatial mapping of dengue fever spread in SriLanka (Lwin et al., 2019) to disease outbreaks surveillance in Germany (Krause et al., 2007). The positive impact of such systems on the management of chronic diseases was also assessed (Roshanov et al., 2011; Michael et al., 2013). For the specific context of epidemiological studies of parasitic diseases, Gray et al (2009) presented a DSDM designed to process data collected during field-based surveillance of the transmission of bovine Schistosoma japonicum in China (Gray et al., 2009). The DSDM consists in a database with a multi-user interface developed using Microsoft Access, VBA and SQL. The authors demonstrated the relevance of digitizing the data collection and management processes in leveraging the data quality and its impact on the study reliability (Gray et al., 2009). Implementing similar DSDM for the collection and the management of CL-related data is of high importance in addressing the under reporting of CLcases worldwide and the mapping of the disease spread and burden.

The present study is focused on the development of a DSDM specific to CL cases, called Lesionia. It was initially developed for the needs of the PEER518 project consortium, but can easily scale-up to other consortia and cutaneous diseases. The project aims at developing speciesspecific point of care diagnostics for CL. These molecular tools will be tested and validated on clinical samples collected in three countries of the MENA region, namely Tunisia, Morocco and Lebanon. The project consortium includes nine institutions based in five countries: Tunisia, Morocco, Lebanon, Mali and the USA. The central node of the consortium is the laboratory of Molecular Epidemiology and Experimental Pathology Applied to Infectious Diseases (MEEPlab) at Institut Pasteur de Tunis (IPT). Lesionia consists of a database and a web-based user interface (WUI). It offers a digital platform for clinical and epidemiological data management for 
CL cases across multiple institutions and countries, along with a tool for experimental data handling and analysis.

\section{Implementation \& Results}

\section{Data collection}

In the frame of the PEER518 project, patient recruitment is taking place in five sites located in four countries (Supplementary table S1). Patients are informed and their written consent is obtained. Clinical samples from patients suspected for cutaneous leishmaniasis are collected, anonymously coded then transferred to the MEEP-lab at IPT, along with related data. At the MEEP-lab, species identification is performed or confirmed using different molecular techniques, the results are collected and the samples are stored. Classically, clinicians and practitioners use a simple form that differs from one hospital to another. Prior to implementing the database and in order to harmonize the data collection process within the consortium and enhance data quality, a discussion took place between clinicians, biologists and bioinformaticians. It aimed at defining the data to be collected from patients and respective samples and how it was to be used. This led to the definition of a questionnaire form (Figure 1). It included patient demographic data, residency and travel history, environmental data related to the notion of insect bite and the animals encountered in close proximity, clinical data including previous treatments if any, data on the clinical aspect of the lesion(s) suspected to be CL (CL could have different presentations), the sampling method and the diagnosis results. The latter include direct examination performed at the sampling site and a selection of molecular tests (PCR ITS, qPCR, RPA-LF) that are further performed at the MEEP-lab for species identification. The questionnaire was conceived with respect to the best practices of data collection (Burguess, 2001; Kelley et al., 2003). First, the questions' order and phrasing were established with practitioners to ensure they can fluently go through them during interviews. We verified that interrogators shall understand the questions consistently. Response fields were reduced to checkboxes with pre-coded responses with a supplementary field for a potential non pre-coded response when it applies. In fact, these formats minimize handwriting, and subsequently spelling and transcription errors during data entry into the database. Units were specified whenever necessary and a canonical format was provided for dates. 
1. Date of consultation

2. Hospital

3. Physician :

4. Sampler :

\begin{tabular}{|c|c|c|}
\hline \multicolumn{3}{|l|}{ Personal Data (to be hidden) } \\
\hline 1. First Name: & 2.Last name : & 3. Medical file number \\
\hline 4. Phone number: & 5.Adress : & \\
\hline \multicolumn{3}{|l|}{ Patient Data } \\
\hline 1.Country: & 2.Governorate: & 3.City : $\quad \square$ Urban $\square$ Rural \\
\hline 4. Nationality / Origin: $\square \mathrm{TN} \square \mathrm{LB}$ & $\square \mathrm{SY} \square \mathrm{MA} \square \mathrm{DZ} \square$ Other, specify: & 5.Notion of bite: $\square$ Yes $\square$ No \\
\hline 6. Gender: $\quad \square \mathrm{F} \square \mathrm{M}$ & 7. Date of birth $\ldots-I_{\ldots} I_{-\ldots}$ & 8.Consent: $\quad \square$ Yes $\square$ No \\
\hline
\end{tabular}

\section{Epidemiological Data}

\section{Travel during the last 12 months before the onset of the lesion:}

\begin{tabular}{|c|c|c|c|c|c|c|}
\hline Date of travel & Duration & Country & Governorate & City & $\begin{array}{c}\text { Urban/ } \\
\text { Rural }\end{array}$ & $\begin{array}{c}\text { Notion of bite } \\
\mathrm{Y} / \mathrm{N}\end{array}$ \\
\hline$-{ }_{-} /{ }_{-} / I_{-}---$ & $-\mathrm{D}_{--} \mathrm{M}_{--} \mathrm{Y}$ & & & & & \\
\hline$-{ }_{--} / \ldots-I_{----}$ & $-\mathrm{D}_{--} \mathrm{M}_{--} \mathrm{Y}$ & & & & & \\
\hline$-{ }_{--} / \ldots-I_{----}$ & $-{ }_{--} \mathrm{D}_{--} \mathrm{M}_{--} \mathrm{Y}$ & & & & & \\
\hline$-{ }_{--} / \ldots-I_{----}$ & ${ }_{--} \mathrm{D}_{--} \mathrm{M}_{--} \mathrm{Y}$ & & & & & \\
\hline
\end{tabular}

2. Presence of animals in the entourage: $\square$ Yes $\square$ No if Yes, specify : $\square$ Bovines $\square$ Ovines $\square$ Caprines $\square$ Camels $\square$ Equids Hares $\square$ Cats $\square$ Dogs $\square$ Foxes $\square$ Other Canids $\square$ Sand rats $\square$ Meriones $\square$ Other rodents $\square$ Gondis $\square$ Hedgehogs $\square$ Bates $\square$ Hyrax $\square$ Other

3. Persons in the entourage who had similar cutaneous lesions or has been diagnosed for Leishmaniasis? : $\square$ Yes $\square$ No if Yes, specify Relationship: $\square$ Family $\square$ Neighbor $\square$ Colleague

4. Place of contact: $\square$ Household $\square$ Neighborhood $\square$ Workplace $\square$ On travel

History of treatment given w/o diagnosis, before the present visit to the center / hospital

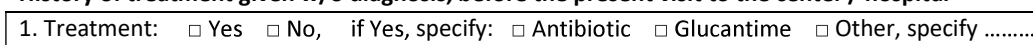

Prescribed for Leishmaniasis: $\square$ Yes $\square$ No

\begin{tabular}{|c|c|c|}
\hline 2. Date of start of the treatment & 3. Duration of treatment & 4. Injection number (Glucantime) \\
\hline
\end{tabular}

\section{History of diagnosis of the present lesions}

1. Before this visit, parasitological/molecular diagnosis has been done $\square$ Yes $\square$ No, if Yes, specify how:

1. Before this visit, parasitological/molecular diagnosis has been done $\square$ Yes $\square$ No, if
$\square$ Direct examination $\square$ Positive $\square$ Negative Date __- /__ /__-_ Laboratory:

$\square \mathrm{PCR} \quad \square$ Positive $\square$ Negative Date $\ldots-I_{-} / \ldots$

2. Did you receive treatment after such diagnosis? $\square$ Yes $\square$ No, if Yes, specify:

$\begin{array}{llll}\square \text { Antibiotic } & \text { Duration: }:-D_{--} M_{---} Y & \text { Posology: } & \text { Administration route: } \\ \square \text { Glucantime } & \text { Duration : }{ }_{--} D_{--} M_{--} Y & \text { Posology: } & \text { Administration route: }\end{array}$

$\square$ Other, specify ............ Duration : ${ }_{--} \mathrm{D}_{--} \mathrm{M}_{---} \mathrm{Y} \quad$ Posology: Administration route:

\section{Interrogator}

First name
Clinical Data

1. Patient's clinical state: $\square$ High blood pressure $\square$ Diabetes $\square$ Allergy $\square$ Pregnancy $\square$ NtR $\left(^{*}\right) \square$ Other, specify ......... \begin{tabular}{|l|l|l|}
\hline 2. Lesion site: $\square$ Face $\square$ Upper limbs $\square$ Lower limbs $\square$ Trunk $\square$ Other, specify............... & 3. Number of lesions: .
\end{tabular}

\section{Sample Data: (Please indicate data relative to the sampled lesion)}

1.Date of onset of the lesion $\ldots-I_{-} / \ldots \ldots$ 2.Lesion site:

3. Lesion description: $\square$ Ulcerative crusty $\square$ Dry $\square$ Wet $\square$ Surrounded by a hyperpigmented rim $\square$ Nodules pseudosporotrichoïdes $\square$ Pseudotumoral $\square$ infected $\square$ Surrounded by an erythematous eruption

$\square$ Other, specify ...

4. Sampling method $\square$ Scrapping $\square$ Aspiration $\square$ Biopsy $\square$ Dental broch $\square$ Swab $\square$ other: specify ...............

5.Sample support $\square$ TE $\square$ Slide $\square$ Saline $\square$ RNA later $\square$ Filter paper

6. Preliminary results: Direct examination $\square$ Positive $\square$ Negative

Abundance on the smear: $\square_{++++++}$口+++++ $\square++++\square+++\square++~ \square+$

\begin{tabular}{|c|c|c|c|}
\hline & & & \\
\hline Antib & Duration :__ $\mathrm{D}_{-} \mathrm{M}_{--} \mathrm{Y}$ & Posology: & Administration route: \\
\hline Glucantime & ${ }_{-} D_{-} \mathrm{M}_{---} \mathrm{Y}$ & Posology: & Administration route: \\
\hline Other, specify & Duration: & Posology: & Administration route: \\
\hline
\end{tabular}

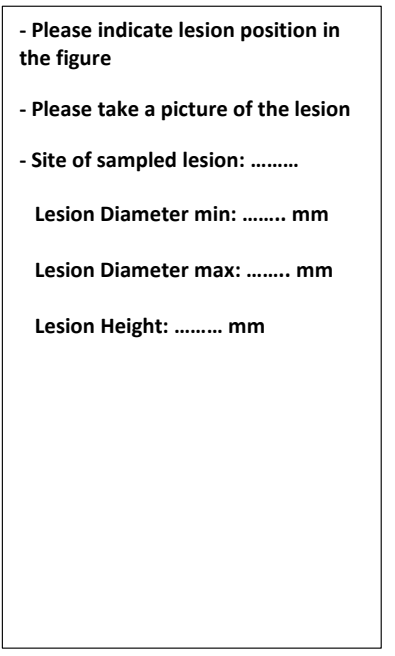

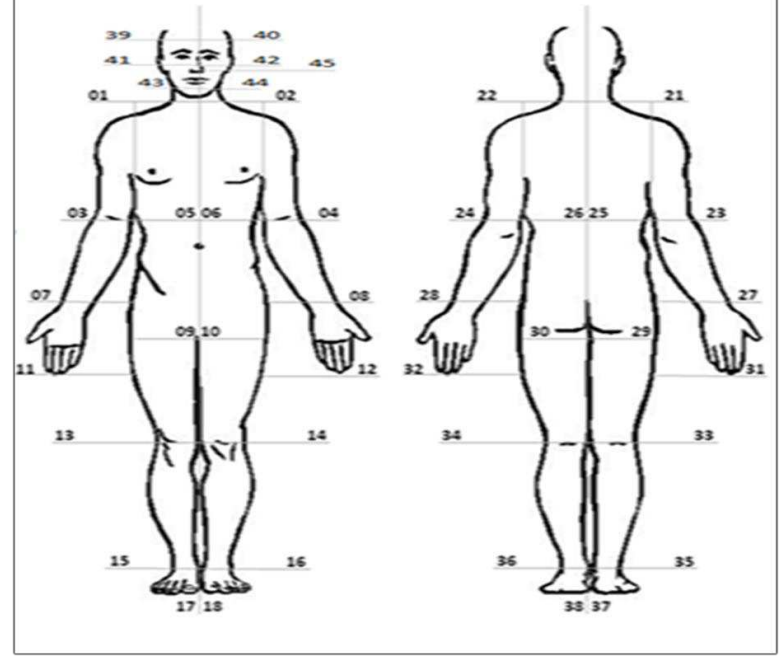

Diagnosis

Molecular tests : please indicate (+ve / -ve )

\begin{tabular}{|c|c|c|c|c|c|c|}
\hline \multirow[t]{2}{*}{ Molecular tests } & \multirow{2}{*}{$\begin{array}{l}\text { Molecular } \\
\text { detection }\end{array}$} & \multicolumn{4}{|c|}{ Species identification } & \multirow[b]{2}{*}{$\mathrm{N} / \mathrm{A}$} \\
\hline & & L. major & L. infantum & L. tropica & Other & \\
\hline \multicolumn{7}{|l|}{ PCR ITS } \\
\hline qPCR & & & & & & \\
\hline RPA-LF & & & & & & \\
\hline
\end{tabular}

$\left({ }^{*}\right)$ Nothing to Report. 
Figure 1. The questionnaire form used by clinicians and practitioners in the partner institutions.

\section{System architecture}

The DSDM implemented for the need of the consortium partners was called Lesionia. It contains multiple components as shown in figure 2. First, the database was designed and implemented using MySQL (Supplementary figure S1). It included data collected by the clinicians, data relative to biobanking in the MEEP-lab and data on the different users that shall use the system, their affiliations and functions. Users have unique login and passwords, provided by the system administrator for a secure connection. The back-end communicates with the database which is hosted on a LEMP server - a variation of the LAMP software bundle that uses Nginx rather than Apache, also known as LNMP (Linux, Nginx, MySQL, PhP). The front-end is designed to be a web-based user interface (WUI) accessible from any web browser. The WUI communicates with a shiny-server hosted on the LEMP server which submits a data request to the database. All servers and the database are physically hosted at the MEEP-lab (IPT). Both the front-end and back-end were developed using R. A list of all packages and dependencies were listed in the project repository on Github (https://github.com/Harigua/LEISIApp ).

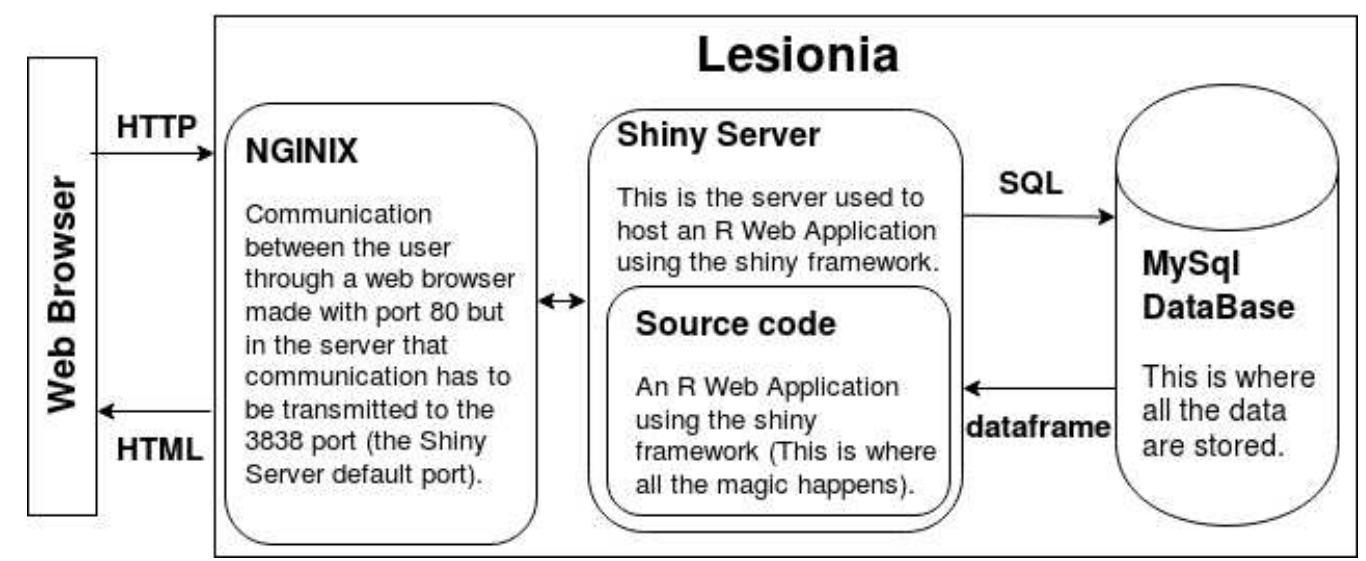

Figure 2. Lesionia architecture, including the database, the front-end and back-end components.

\section{The web user interface (WUI)}

We developed the WUI using the Shiny framework implemented in R. It is accessible through www.lesionia.pasteur.tn/. It has a homepage that contains the login form and information on the PEER518 project, the consortium and how to contact the system administrator and/or the research team (Figure $3(\mathrm{a})$ ).

Using a username and a password previously validated by the system manager, users can access four sections (Figure 3 (b)): (i) Data management, (ii) Data entry, (iii) Data viewer, (iv) Data analysor. The WUI includes a header that contains the application name 'Lesionia', the name of the currently logged in user and a 'logout' button. The 'Lesionia' button allows the user to go back to the homepage from anywhere during the use of the application and thus to switch 
between sections.

The "Data Management" section has a restrictive access to the super user(s). It permits to add new user(s), edit existing user(s) or delete user(s). It also permits deleting or downloading data using filters.

"Data entry" is the section that includes all the digital forms and interfaces to enter new data or update existing data. A double-entry system was implemented to minimize the rate of discrepancy. The first entry consists in creating a unique ID for a patient. To continue entering the data, the user has to re-enter the unique ID, and an automatic check of the concordance of IDs is performed. If the second entry is not correct, the user will know and correct it accordingly. If the first entry is not correct, the user should report this discrepancy using a dedicated field. Based on the discrepancy report, the system manager could intervene. For each section in the questionnaire, a digital form exists in Lesionia. These forms present multiple fields, out of which those that are mandatory are tagged with an asterisk. Missing data fields can be left blank. Default values are beforehand assigned to indicate that these fields correspond to missing data. These default values are "N/A" or “- 1 ” for data of type character or integer, respectively. Missing data with type date are set to 01-01-1900. This is important for the forthcoming data analysis steps and for the assessment of the data collection procedures.

All data in the questionnaire form can be entered in the corresponding tabs of Lesionia interface (Figure 3 (c)).

"Data viewer" allows the user to visualize, search and browse all the data within the tables of the database. It also provides statistics on patients recruitment flow, partitions according to nationality, gender and age classes and raw data visualization and download.

"Data analysor" contains sections dedicated to data analysis, including: (i) General statistics, (ii) Correlation between the SPECIES variable and any other chosen variables, (iii) Regression (Linear model, Chi square) and (iv) Multiple Correspondence Analysis (MCA). Almost all the analyzes are dynamic and can be customized by choosing the variables to be considered. All resulting graphs and figures can be directly downloaded.

In order to assess the reliability of the "Data analysor" functionality, we simulated data for 3000 patients. For some data fields, specific distributions were applied according to preliminary statistics provided by our clinician partners (Supplementary Table S2). Otherwise, uniform distributions were applied. The sample size had no effect on the speed of the application. The statistical analyses obtained with the "Data analysor" were consistent with the sample distributions, thus confirming that all statistical tests were correctly implemented (Figure 4). Lesionia was implemented as an open software accessible through the following link (https://github.com/Harigua/LEISIApp/). To make this software further accessible to the scientific community willing to use it as is or customize it, we developed a user guide on how to install it on different operating systems (Linux, Mac and windows). It can either be installed locally as a desktop application or as a web-based application (Supplementary file S1). It presents the advantage of: (i) error checking during data entry, (ii) ability to report discrepancies 
to the system administrator, (iii) a built-in statistical analysis tool and (iv) data fields are easily expandable as for the case of biological tests, studied species, etc. On the other hand, it presents some disadvantages, namely, (i) it is closely related to our questionnaire and further development is needed to use it for other diseases and (ii) no logs of data edits are saved for the actual version, but this can be included.

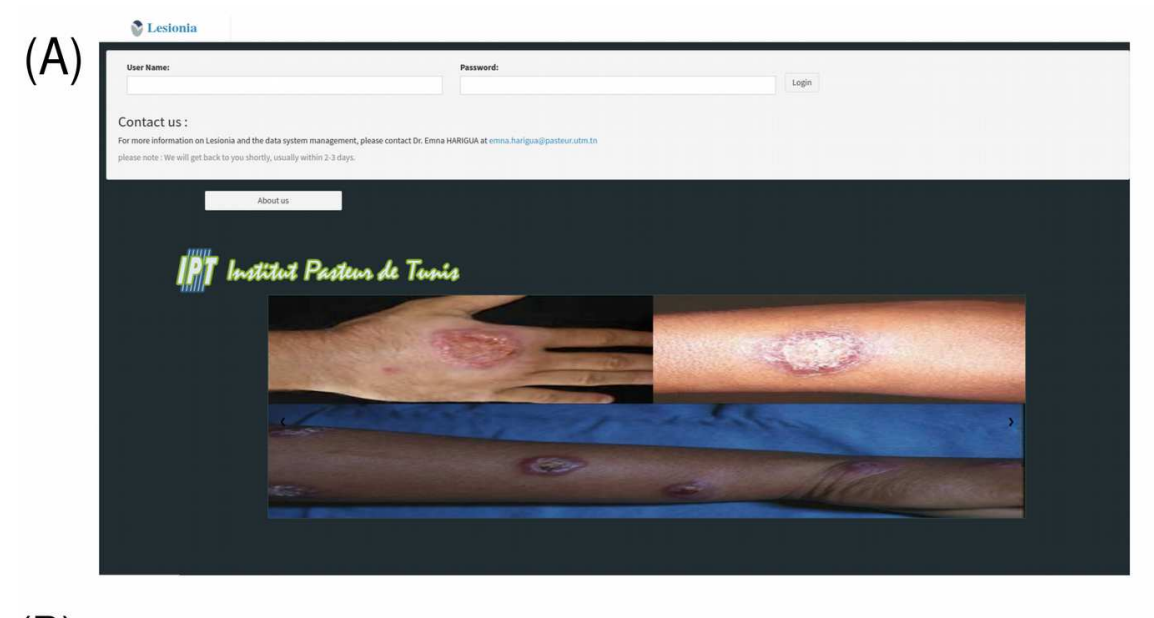

(B)

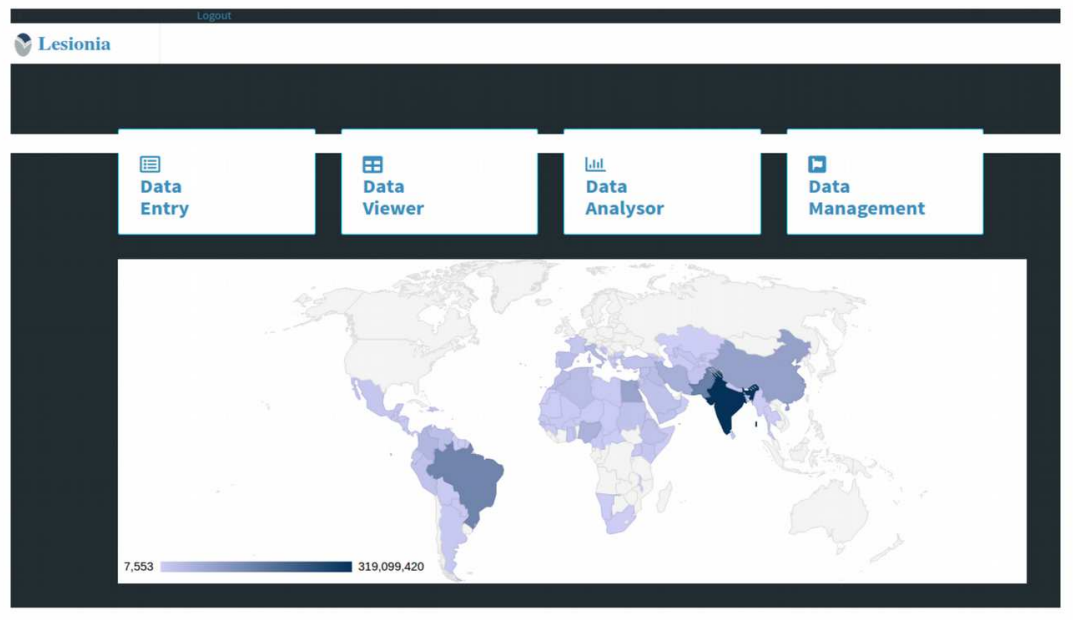

(C)

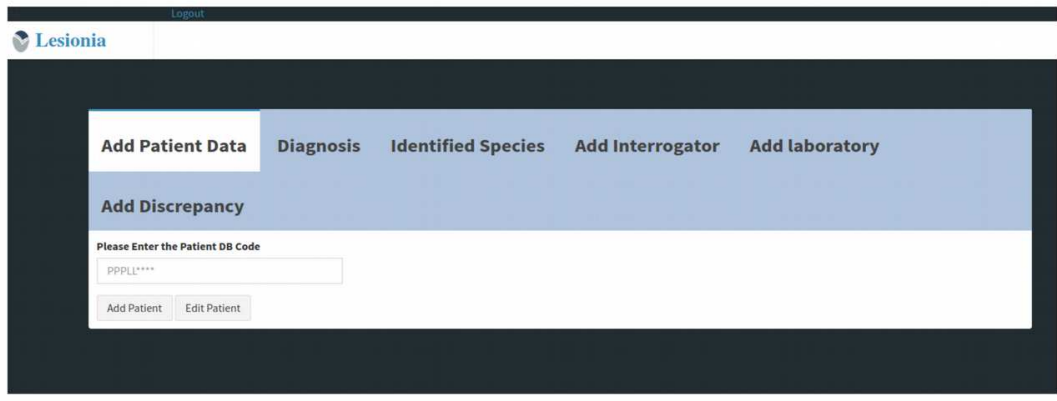

Figure 3. Lesionia web-based user interface. (A) The homepage appearing by default through the link www.lesionia.pasteur.tn/ . (B) The homepage that appears after logging in. (C) The interface behind the "Data entry" section. It includes all tabs necessary for data entry. 

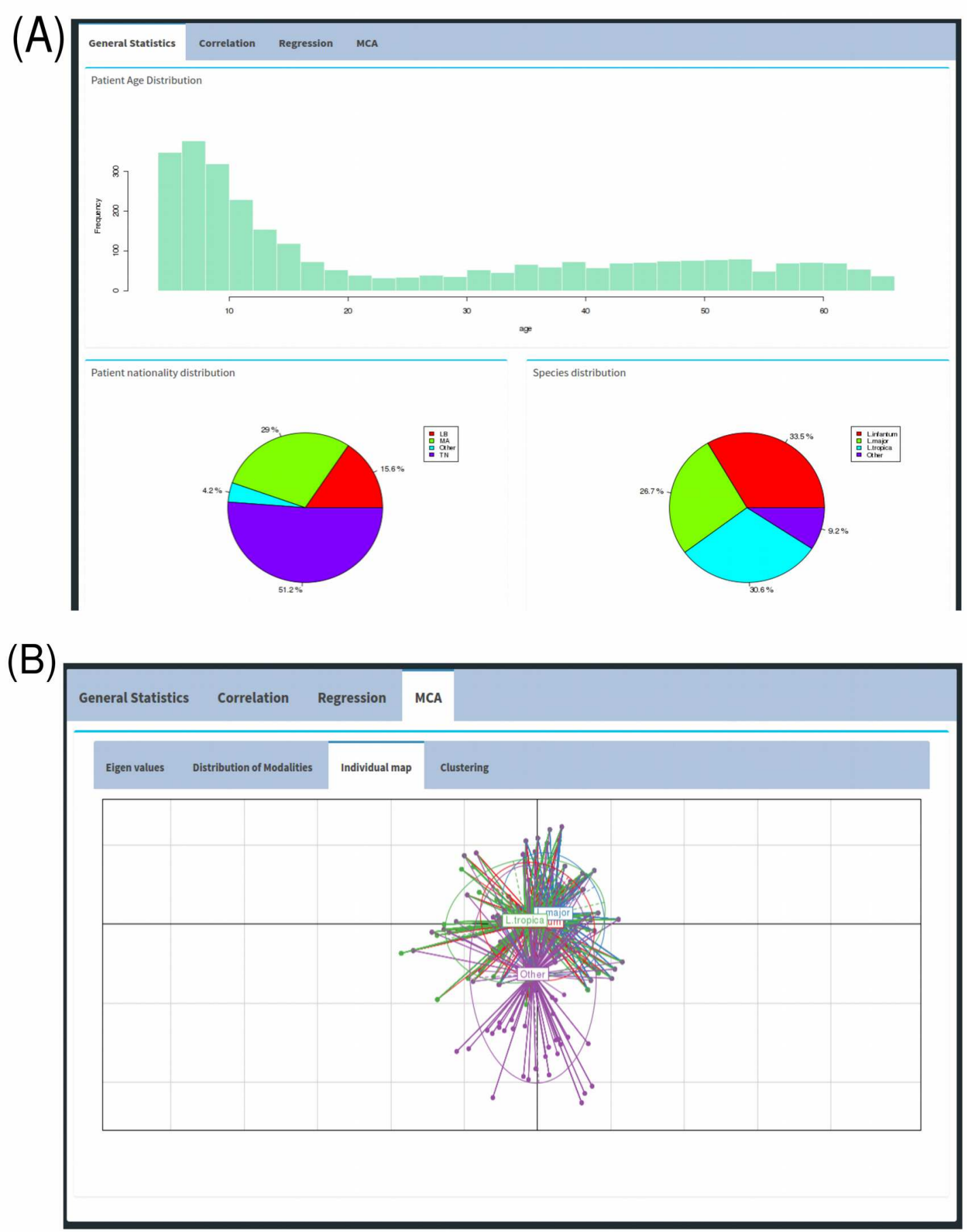

Figure 4. The built-in data analysor of Lesionia. (A) General statistics results obtained for the simulated data on a 3000-individual sample. (B) Multiple correspondence Analysis (MCA) obtained for the simulated sample. Positive samples present different individual maps as compared to negative samples with no discriminative power between the different species, which is in concordance with the simulated data conditions. 


\section{Discussion}

The positive impact of implementing digital systems for data management (DSDM) has been now thoroughly demonstrated in different fields of biomedical sciences (Roshanov et al., 2011; Xue-Jaun et al., 2018; Ngwatu 2018). DSDM provide sustainable systems for data management, reliable tools for data collection and efficient platforms for descriptive and predictive analytics (Lwin et al., 2019; Bell et al., 2018). As research data are often specific to their collection context and research objectives, there are limited possibilities for tools repurposing. Thus, implementing dedicated systems is often a requisite. In this context, a dedicated DSDM was implemented in a chinese community with the objective of embedding epidemiological data collection on Schistosoma japonicum spread during onsite intervention processes (Gray et al., 2009). This system enabled researchers to produce good-quality data while maximizing the rate of digitizing data collection and reducing the costs of data cleaning. It assures quality of the data through a double-entry check and quality control metrics. It included a built-in statistical analysis functionality and an automated code generator. On the other hand, the system presents no interoperability and is restricted to Microsoft Windows operating system. In this specific context, we herein present Lesionia as an open source pilot software for the collection, management and analysis of data relative to patients with cutaneous diseases such as CL. Lesionia has the advantage of allowing multi-users data entry even for a single patient/sample. This is of high importance in smoothing the multi-actors process of data collection from patient recruitment to sample storage and molecular tests performance. This advantage is valuable for the harmonization of the multicentric process of the experimental detection and identification of the CL causing species. Lesionia is also an ethically-sound application that uses anonymous coded data. Through the double-entry check for ID codes, it guarantees a reliable tracking system of data entries. These IDs also connect the digital entries to the paper forms that are kept by the clinicians. These paper support include patients' personal data and clinical records that are confidential and are not shared through the consortium. Thus, they are not included in the database. These confidential data can only be used by the clinicians in case there is a need to contact patients during outbreaks or other sanitary issues. Lesionia also offers a built-in functionality for real-time data analysis. This is one of the most practical features as it permits all users to run sophisticated and various statistical analysis in a user-friendly and coding-free fashion. Thus the users do not need to be experts in scripting or coding to generate the analyses. Another group of researchers recently published Epihak, a prototype of a DSDM developed through a health hackathon in Sri Lanka (Lwin et al., 2019). It has been implemented to study and combat dengue fever using an integrative framework that includes mobile technology. Epihak permitted to digitize hospital forms and enhance track surveillance of dengue fever across Sri Lanka. It presented the advantage of using a mobile application which increases the rate of field-based data collection. The authors demonstrated the impact of mobile technology use on the speed and accuracy of data collection, management and analysis as compared to classical methods that include paper forms and outdated digital applications. Although, Lesionia 
can be accessed through a web browser from a mobile device (phone/pad), the user experience is not optimal. We intend to deploy it in a next step as a mobile application as these technologies facilitate field-based data collection processes. They also permit the access in a very userfriendly fashion to build-in functionalities of the device, such as the camera, the localization and the user identification (Fraser et al., 2012; Lwin et al., 2019). Embedding technological solutions in the governmental and large scale health systems proved to drastically impact disease control and management (Fraser et al., 2012; Bell et al., 2018; Lwin et al., 2019).

\section{Conclusion and future directions}

Lesionia is an innovative application for clinical and epidemiological data management of CL patients. It offers a multi-site and multi-user web-based application which enhances the efficacy of the data sharing process. It was implemented using interoperable languages which makes it usable on different operating systems. As part of implementing reliable and sustainable data management systems in our lab and institute, Lesionia was also used as part of an institutional clinical investigation project at IPT. It can scale up to a more widely used system in managing and reporting cutaneous diseases in health care centers across the country and the region. Also, it can be reused for other multicentric trials or projects.

\section{Ethics approval and consent to participate}

Ethical approval was obtained from respective IRBs to each sampling site, under the references 2016/24/I/LRIPT04 of IPT-Tunisia, 51/17 of the "Comité d'éthique pour la recherche biomédicale, Faculté de Médecine et de Pharmacie-Université Mohammed V-Rabat” in Morocco and INV-2017-324 of the Institutional Review Board-Rafik Hariri University Hospital in Lebanon.

\section{Consent for publication}

Not applicable

\section{Competing interests}

The authors declare that they have no competing interests

\section{Availability of data and materials}

The datasets generated and analysed during the current study are available in the public repository on Github https://github.com/Harigua/LEISIApp/

\section{Funding}

This work was supported by the NAS-USAID PEER programme - cycle 5 (PEER 518). It also received partial support from the initiative of Clinical Investigation Centre (CIC) at Institut Pasteur de Tunis (CIC2016IPT02) and the Ministry of Higher Education and Research in Tunisia 
(LR16IPT04). The funders had no role in study design, data collection and analysis, decision to publish, or preparation of the manuscript.

\section{Authors' contributions}

EHS, YS, IBA and IG conceived the research.

EHS, YBS and OS conceived and designed the database.

EHS, YBS and MH developed the WUI.

MH, HS, HC, IM, ZM, NH, AFM and ML provided user feedback to optimize the questionnaire format and/or to mitigate the digital system's setbacks.

EHS and MH assessed the system's viability and performances.

EHS drafted the original manuscript.

EHS,YS, OS, IBA and IG reviewed and edited the manuscript.

All authors read and approved the final manuscript.

\section{Acknowledgements}

The authors acknowledge the contribution of all partners within the PEER518 consortium, namely Pr. Kalthoum Kallel, Dr. Aicha Kallel, Pr. Slah Bel Haj, Dr. Imen Khammeri, Dr. Adil El Hamouchi, Mr. Othmane Daoui, Dr. Pierre Abi Hanna, Dr. Oussaima El Dbouni, Dr. Bourama Traore, Pr. Seydou Doumbia, Pr. Rhea N. Coler, Pr. Steven Reed, for their recommendations during the setup of the project and the annual meetings. 


\section{References}

1. Alam E, Abbas O, Moukarbel R, Khalifeh I. Cutaneous leishmaniasis: an overlooked etiology of midfacial destructive lesions. PLoS neglected tropical diseases. 2016 Feb 10;10(2):e0004426.

2. Ali M, Park JK, Von Seidlein L, Acosta CJ, Deen JL, Clemens JD. Organizational aspects and implementation of data systems in large-scale epidemiological studies in less developed countries. BMC Public Health. 2006 Dec;6(1):86.

3. Bailey F, Mondragon-Shem K, Hotez P, Ruiz-Postigo JA, Al-Salem W, Acosta-Serrano A, Molyneux DH. A new perspective on cutaneous leishmaniasis-Implications for global prevalence and burden of disease estimates. PLoS neglected tropical diseases. 2017 Aug 10;11(8):e0005739.

4. Bell D, Gachuhi N, Assefi N. Dynamic Clinical Algorithms: Digital Technology Can Transform Health Care Decision-Making. The American journal of tropical medicine and hygiene. 2018 Jan 10;98(1):9-14.

5. Bennis I, De Brouwere V, Belrhiti Z, Sahibi H, Boelaert M. Psychosocial burden of localised cutaneous Leishmaniasis: a scoping review. BMC Public Health. 2018 Dec;18(1):358.

6. Burgess TF. Guide to the Design of Questionnaires. A general introduction to the design of questionnaires for survey research. 2001 May;30(4):411-32.

7. Eisen L, Coleman M, Lozano-Fuentes S, McEachen N, Orlans M, Coleman M. Multidisease data management system platform for vector-borne diseases. PLoS neglected tropical diseases. 2011 Mar 29;5(3):e1016.

8. $\quad$ Fraser HS, Thomas D, Tomaylla J, Garcia N, Lecca L, Murray M, Becerra MC. Adaptation of a web-based, open source electronic medical record system platform to support a large study of tuberculosis epidemiology. BMC medical informatics and decision making. 2012 Dec;12(1):125.

9. Gray DJ, Forsyth SJ, Li RS, McManus DP, Li Y, Chen H, Zheng F, Williams GM. An innovative database for epidemiological field studies of neglected tropical diseases. PLoS neglected tropical diseases. 2009 May 26;3(5):e413.

10. Jajosky RA, Groseclose SL. Evaluation of reporting timeliness of public health surveillance systems for infectious diseases. BMC public health. 2004 Dec;4(1):29.

11. John Roberts R, Musick BS, Olley B, Hall KS, Hendrie HC, Oyediran AB. Data management in a longitudinal cross-cultural study. Statistics in medicine. 2000 Jun 15;19(1112):1645-9.

12. Kelley K, Clark B, Brown V, Sitzia J. Good practice in the conduct and reporting of survey research. International Journal for Quality in health care. 2003 May 1;15(3):261-6.

13. Krause G, Altmann D, Faensen D, Porten K, Benzler J, Pfoch T, Ammon A, Kramer MH, Claus H. SurvNet electronic surveillance system for infectious disease outbreaks, Germany. Emerging Infectious Diseases. 2007 Oct;13(10):1548. 
14. Lwin MO, Sheldenkar A, Panchapakesan C, Ng JS, Lau J, Jayasundar K, Horathalge K, Rathnayake VS, Crawley AW, Wimalaratne P. Epihack Sri Lanka: development of a mobile surveillance tool for dengue fever. BMC medical informatics and decision making. 2019 Dec;19(1):111.

15. Ma J, Otten M, Kamadjeu R, Mir R, Rosencrans L, McLaughlin S, Yoon S. New frontiers for health information systems using Epi Info in developing countries: Structured application framework for Epi Info (SAFE). International journal of medical informatics. $2008 \mathrm{Apr}$ 1;77(4):219-25.

16. Michael BD, Geleta D. Development of ClickClinica: a novel smartphone application to generate real-time global disease surveillance and clinical practice data. BMC medical informatics and decision making. 2013 Dec;13(1):70.

17. Ngwatu BK, Nsengiyumva NP, Oxlade O, Mappin-Kasirer B, Nguyen NL, Jaramillo E, Falzon D, Schwartzman K. The impact of digital health technologies on tuberculosis treatment: a systematic review. European Respiratory Journal. 2018 Jan 1;51(1):1701596.

18. Pigott DM, Golding N, Messina JP, Battle KE, Duda KA, Balard Y, Bastien P, Pratlong F, Brownstein JS, Freifeld CC, Mekaru SR. Global database of leishmaniasis occurrence locations, 1960-2012. Scientific data. 2014 Sep 30;1:140036.

19. Roshanov PS, Misra S, Gerstein HC, Garg AX, Sebaldt RJ, Mackay JA, Weise-Kelly L, Navarro T, Wilczynski NL, Haynes RB. Computerized clinical decision support systems for chronic disease management: a decision-maker-researcher partnership systematic review. Implementation Science. 2011 Dec 1;6(1):92.

20. Tabbabi A. Review of Leishmaniasis in the Middle East and North Africa. African health sciences. 2019;19(1):1329-37.

21. Thomsen EK, Deb RM, Dunkley S, Coleman M, Foster G, Orlans M, Coleman M. Enhancing decision support for vector-borne disease control programs - the disease data management system. PLoS neglected tropical diseases. 2016 Feb 18;10(2):e0004342. 


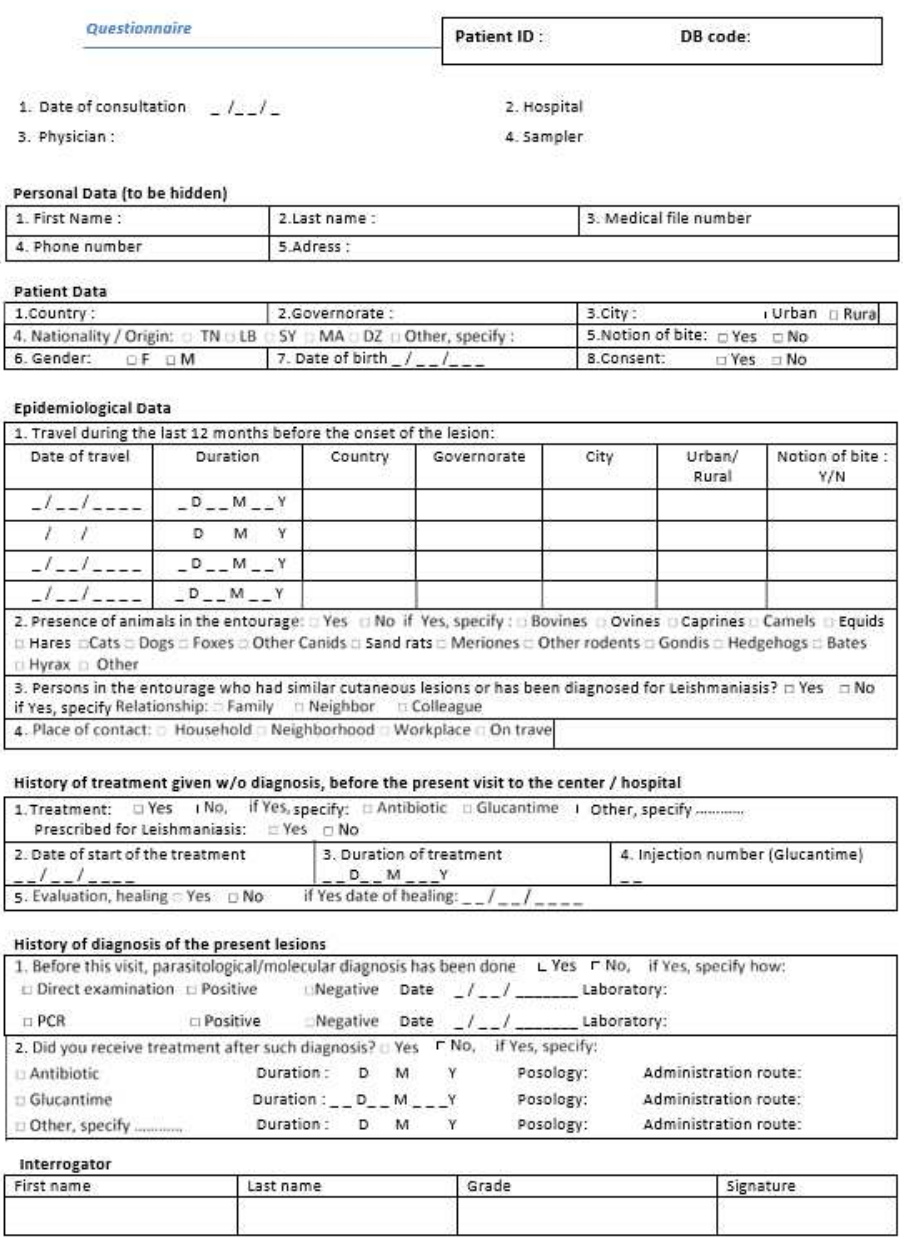

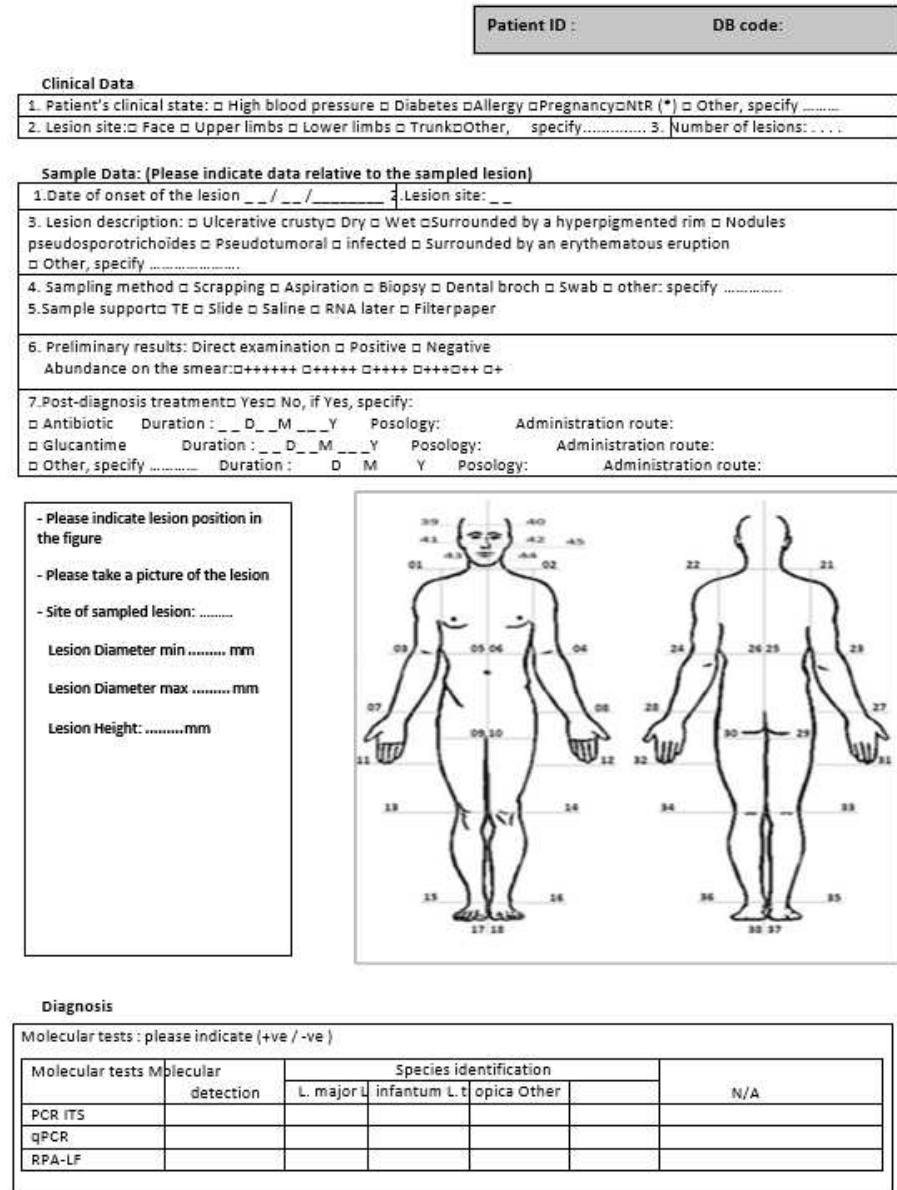

[*]Nothing to Report.

\section{Figure 1}

The questionnaire form used by clinicians and practitioners in the partner institutions.

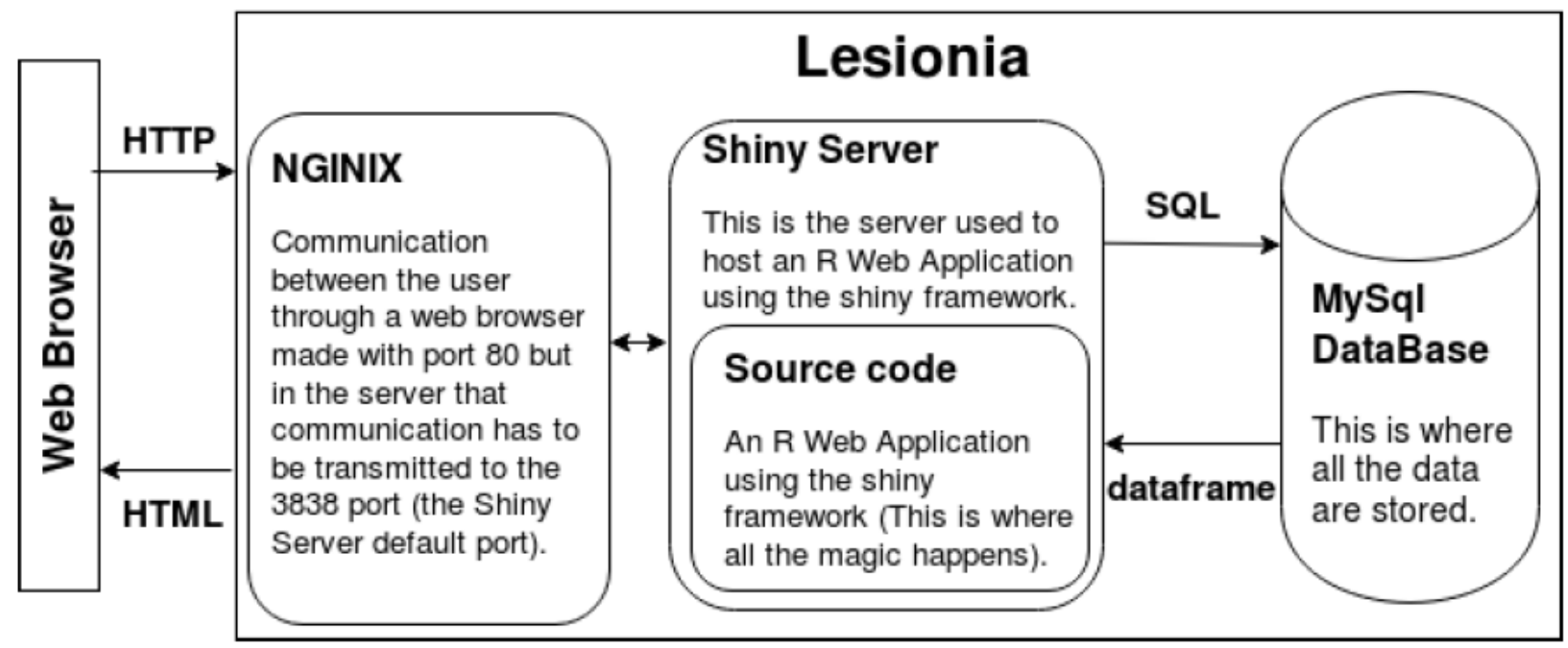


Figure 2

Lesionia architecture, including the database, the front-end and back-end components.

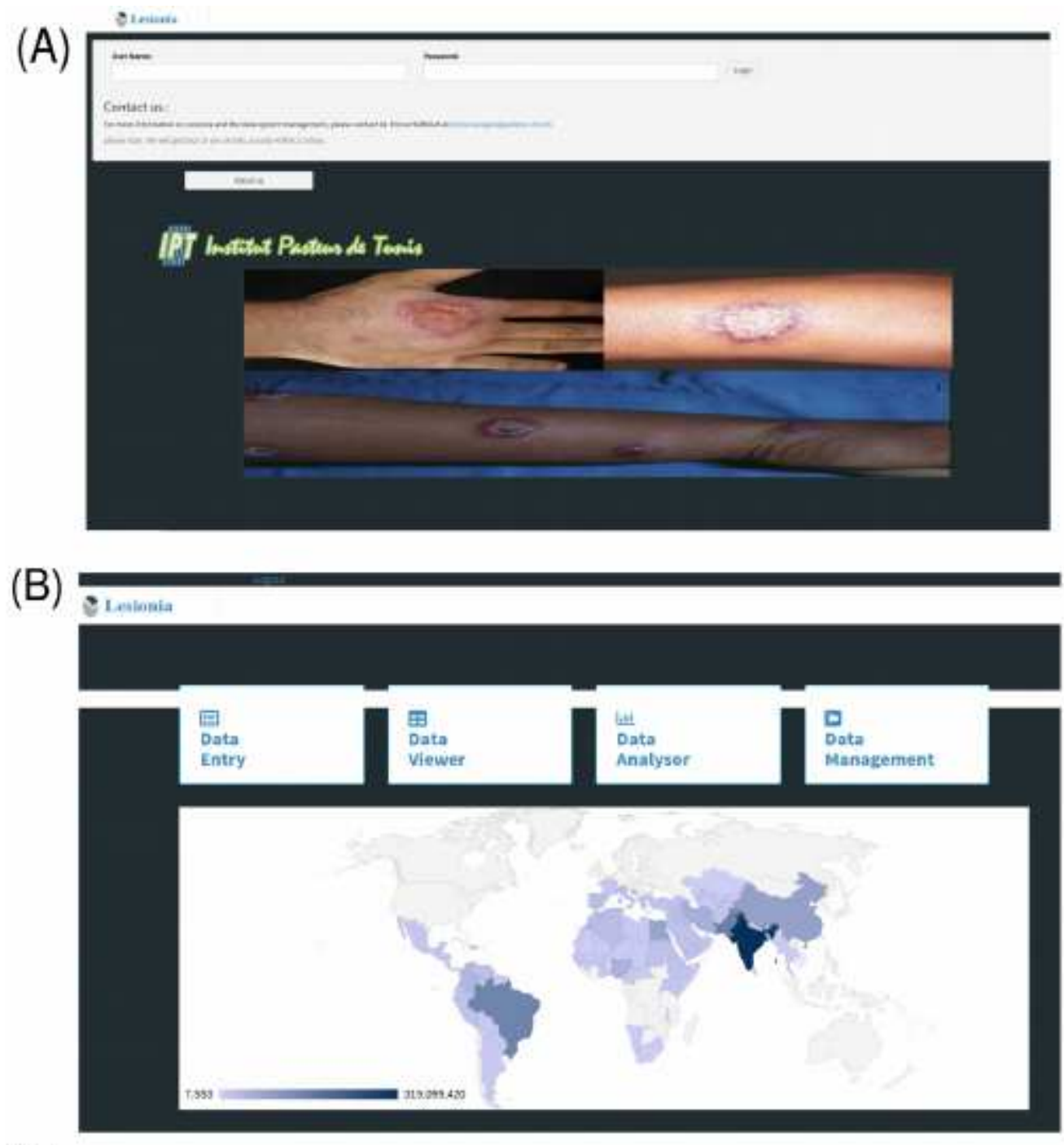

(C)

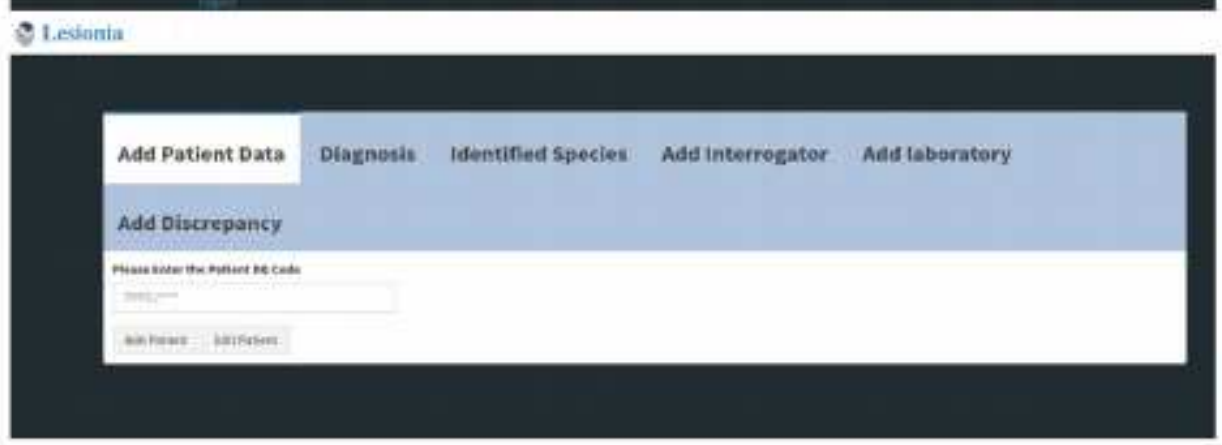

\section{Figure 3}

Lesionia web-based user interface. (A) The homepage appearing by default through the link www.lesionia.pasteur.tn/ . (B) The homepage that appears after logging in. (C) The interface behind the "Data entry" section. It includes all tabs necessary for data entry. 

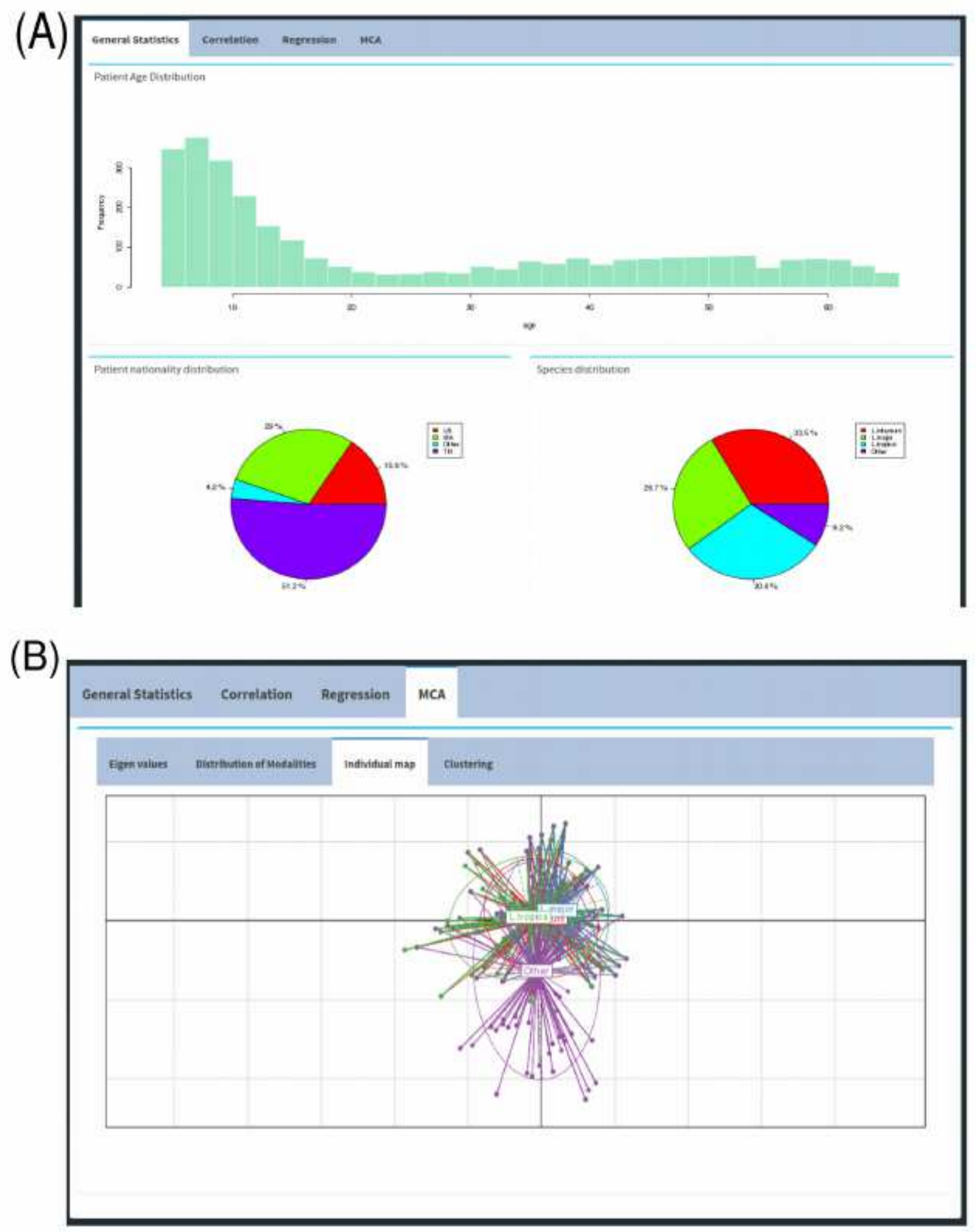

\section{Figure 4}

The built-in data analysor of Lesionia. (A) General statistics results obtained for the simulated data on a 3000-individual sample. (B) Multiple correspondence Analysis (MCA) obtained for the simulated sample. Positive samples present different individual maps as compared to negative samples with no discriminative power between the different species, which is in concordance with the simulated data conditions. 


\section{Supplementary Files}

This is a list of supplementary files associated with this preprint. Click to download.

- HariguaSouiaiSuppMaterial.pdf 